\title{
Changes in height of choroidal melanomas after plaque therapy
}

\author{
David H Abramson, Camille A Servodidio, Beryl McCormick, Daniel Fass, Edith Zang
}

\begin{abstract}
Serial ultrasonic measurements of 82 uveal melanomas treated with brachytherapy plaques (cobalt-60 and iodine-125) and followed up for up to 141 months revealed that no two patients had identical patterns of change. The mean absolute change in tumour height after treatment was $1.8 \mathrm{~mm}$ at six months, $5.6 \mathrm{~mm}$ at 48 months for large tumours, and 0.9 and $1.9 \mathrm{~mm}$ for medium sized tumours. Eighty of the 82 patients fell into one of three patterns of response: 57 patients had a decrease in height after treatment (type D), 13 patients had the same height after treatment (type S), and 10 patients had a progressive increase in height (type I). Life table comparison showed no correlation between survival and location of tumour, sex of patient, size of tumour when treated, or laterality. There was a slight correlation between age and survival. Patients older than 60 died more frequently from metastatic melanoma than those under 60 $(p=0.06)$. Life table analysis showed a significant correlation between tumour regression type and survival. At 48 months the best cumulative probability of survival was in patients with type $D$ ( $88 \%$ alive) compared with those of type I (34\% alive, $\mathrm{p}=0.0004)$.
\end{abstract}

Since Stallard's pioneering work scleral radioactive applicators (brachytherapy) has been used in many ways throughout the world. Cobalt- 60 , iodine-125, iridium-192, and ruthenium-106 have been used. Remarkably little is known about what happens to the ocular tumour in the eye after brachytherapy. Stallard' reported that the majority of melanomas he treated became flat; few other workers have achieved that result. Cruess et al calculated that a 'mean' change in tumour thickness was $20 \%$ reduction at six months, $30 \%$ at 12 months, and then a progressive decrease of $50 \%$ at 54 months. They concluded that the average posterior uveal melanoma treated with cobalt -60 did not regress to a flat scar and that the 'extent and rate of regression' were not reliable indicators of the patient's likelihood of developing metastatic disease. $^{2}$

In the only other series that has been similarly analysed Coleman et al (New York) reported that tumour thickness was not a significant predictor of percentage reduction in height. However, they showed that ultrasonically derived spectral parameters did correlate with regression. ${ }^{3}$ At six months they found that acoustic tissue type (ATT) B had a $29.5 \%$ reduction in height, while ATT E had an $11.4 \%$ reduction in height. At 18 months ATT B had a $53.9 \%$ reduction in height, while ATT E had a $26.4 \%$ reduction in height.
One of the authors of the present paper (DHA) involved in plaque therapy for two decades observed that these papers simplify data and represent an 'average response' that does not agree with all clinical observations. An average response does not accurately reflect what actually happens to ocular tumours after brachytherapy. This could be confusing to a clinician unfamiliar with the responses of treated tumours. Furthermore, there has been little comment in the literature about tumours in the eye that progress after plaque therapy and their significance for the eye and the patient. To answer these questions we retrospectively analysed the tumour height of plaqued melanomas in a series of patients diagnosed by DHA and treated with BM and DF. Follow-up data were available for up to 12 years.

\section{Material and methods}

A retrospective analysis of 83 patients with uveal melanoma treated by one of us (DHA) was performed. The diagnosis of uveal melanoma was made in the usual way by ophthalmoscopy, fluorescein angiography, ultrasonography, and quantative echography, visual fields, and transillumination. The ${ }^{32} \mathrm{P}$ test was done on two patients. No patient had received any prior therapy or a fine needle biopsy. Before treatment all patients were examined for metastastases, and none had been reported for any patient.

Ultrasonic heights of tumours were measured at most follow-up visits. To simplify the groups, plots have been performed at monthly intervals. If the patient had not actually been measured at that time, a mathematical weighted average was made between the two listed visits and this 'averaged' number was used for plotting data. Because of the technical problems associated with repeated ultrasonic measurements, we defined changes in height as subsequent measurements greater than $15 \%$ of the original measurement. Occasionally no ultrasonic measurements were performed, and the clinical estimate of the height was used for that visit. One patient was lost to follow-up.

Metastatic disease was detected by liver function tests, liver spleen scans, needle biopsies (of liver), bone scans, CT scans, magnetic resonance imaging (MRI) scans, and in some cases necropsy.

\section{STATISTICAL METHODS}

The putative association between pairs of categorical variables was analysed through contingency tables and the $\chi^{2}$ statistic. Continuously measured characteristics such as age and tumour size were correlated by the Pearson product moment correlation technique, and the statisti- 
TABLE I Results of treatment

\begin{tabular}{|c|c|c|c|c|c|c|c|c|c|c|c|}
\hline I. & $\begin{array}{l}\text { Patients tre } \\
\text { Number: }\end{array}$ & $\begin{array}{ll}\text { ated }(83) \\
O D & \text { OS } \\
51 & 32\end{array}$ & $\begin{array}{l}\text { Male } \\
53\end{array}$ & $\begin{array}{l}\text { Female } \\
30\end{array}$ & $\begin{array}{l}\text { Age } \\
20-29 \\
4\end{array}$ & $\begin{array}{l}30-39 \\
5\end{array}$ & $\begin{array}{l}40-49 \\
4\end{array}$ & $\begin{array}{l}50-59 \\
13\end{array}$ & $\begin{array}{l}60-69 \\
30\end{array}$ & $\begin{array}{l}70-79 \\
19\end{array}$ & $\begin{array}{l}80-89 \\
7\end{array}$ \\
\hline \multirow[t]{3}{*}{ II. } & \multicolumn{4}{|c|}{$\begin{array}{lll}\text { Tumours treated }(83) & \\
\text { Small } & \text { Medium } & \text { Large } \\
(2 \cdot 0 \mathrm{~mm}- & (3 \cdot 1 \mathrm{~mm}- & (8 \cdot 1 \mathrm{~mm}- \\
3.0 \mathrm{~mm}) & 8.0 \mathrm{~mm}) & 16 \mathrm{~mm})\end{array}$} & \multicolumn{4}{|c|}{$\begin{array}{l}\text { Tumour vertex } \\
\text { Ciliary body } \\
8\end{array}$} & $\begin{array}{l}\text { Equator } \\
8\end{array}$ & & $\begin{array}{l}\text { Posterior equator } \\
53\end{array}$ \\
\hline & 4 & $\begin{array}{l}8 \cdot 0 \mathrm{~mm}) \\
63\end{array}$ & \multicolumn{2}{|c|}{16} & \multicolumn{2}{|c|}{$\begin{array}{l}\text { Cobalt }-60 \\
55\end{array}$} & \multicolumn{2}{|c|}{$\begin{array}{l}\text { Iodine- } 125 \\
28\end{array}$} & & & \\
\hline & \multicolumn{4}{|c|}{$\begin{array}{l}\text { Pairs of variables } \\
\text { Age vs tumour height before treatment } \\
\text { Location vs tumour height before treatment } \\
\text { Age vs location }\end{array}$} & \multicolumn{4}{|c|}{$\begin{array}{l}\text { Correlation coefficient }(R) \\
-0.009 \\
0 \cdot 15 \\
-0.04\end{array}$} & \multicolumn{2}{|c|}{$\begin{array}{l}p \text { Value (t test) } \\
0.93 \mathrm{NS} \\
0.17 \mathrm{NS} \\
0.74 \mathrm{NS}\end{array}$} & \\
\hline
\end{tabular}

cal significance of the correlation coefficients was tested by the $t$ test to estimate the probability that $\mathbf{r} \neq 0$.

Linear regression analysis was employed to test and quantify. the linear component of the relationship between pre- versus post-treatment tumour size at one, two, and three years followup.

The relationship between tumour height at various times of follow-up and the change in tumour height following treatment versus age, survival status, and pretreatment tumour size was evaluated through analysis of covariance. In these models the post-treatment tumour height and the change in tumour height after treatment represented the dependent variable; pretreatment tumour category (small, medium, and large) and survival status were the categorical independent variables; and age was the covariate.

The correlation of various continuous and categorical patient characteristics with survival was analysed by the Cox regression model, ${ }^{4}$ followed by the likelihood ratio test. For univariate survival we used the Kaplan-Meier product limit estimate, ${ }^{5}$ and the statistical significance of the differences between survival distributions was tested through the logrank summary $\chi^{2}$ test. ${ }^{6}$ The distribution of continuous variables was normalised whenever necessary to meet the assumption of specific analytical techniques.

\section{Results}

Results are summarised in Table I.

TABLE II Mean change in tumour height following treatment

\begin{tabular}{|c|c|c|c|c|}
\hline \multirow{2}{*}{$\begin{array}{l}\text { Pretreatment } \\
\text { tumour size }\end{array}$} & \multicolumn{4}{|c|}{ Mean change (in mm) after treatment at } \\
\hline & $6 m o$ & $12 \mathrm{mo}$ & $24 m o$ & $48 \mathrm{mo}$ \\
\hline \multicolumn{5}{|c|}{ I. Actual change (in mm) } \\
\hline Small & $0 \cdot 1$ & $0 \cdot 1$ & - & - \\
\hline Medium & 0.9 & 1.4 & 0.8 & 1.9 \\
\hline Large & & & $3 \cdot 5$ & 5.6 \\
\hline \multicolumn{5}{|c|}{$\begin{array}{l}\text { Absolute decrease in height was most pronounced in large } \\
\text { tumours. }\end{array}$} \\
\hline \multicolumn{5}{|c|}{$\begin{array}{l}\text { The average monthly decrease in height for the first } 48 \text { months } \\
\text { was } 0.5 \text { for the large tumours and } 0.2 \mathrm{~mm} \text { for medium tumours }\end{array}$} \\
\hline \multicolumn{5}{|c|}{ II. Percentage of pretreatment height } \\
\hline Small & 101 & 100 & - & - \\
\hline Medium & 88 & 76 & 87 & 76 \\
\hline Large & 86 & 77 & 71 & 49 \\
\hline \multicolumn{5}{|c|}{$\begin{array}{l}\text { The percentage decrease in tumour height was most pronounced } \\
\text { in large tumours }\end{array}$} \\
\hline \multicolumn{5}{|c|}{ III. Monthly actual change (average) } \\
\hline Small & $0 \cdot 1$ & & - & - \\
\hline Medium & 0.07 & $0 \cdot 12$ & 0.07 & 0.2 \\
\hline Large & $0 \cdot 1$ & $0 \cdot 23$ & $0 \cdot 3$ & $0 \cdot 5$ \\
\hline
\end{tabular}

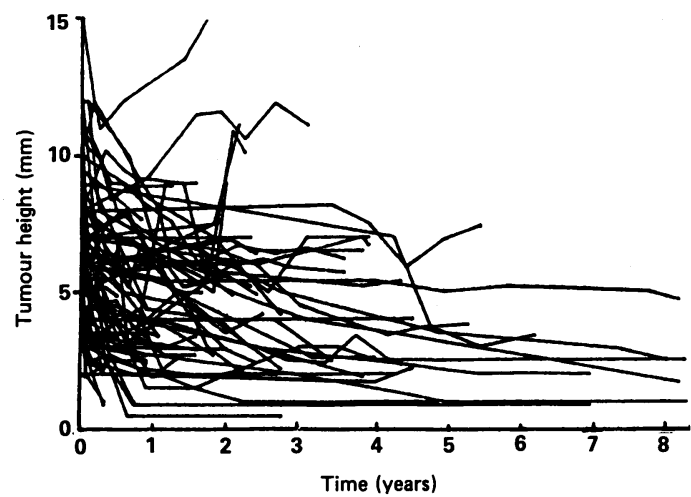

Figure 1: Changes in height of tumours in each of the 83 patients.

\section{PATTERNS OF CHANGE AND METASTASES}

The changes in tumour height over time are plotted in Figure 1, and see Table II. No two tumours had exactly the same pattern of change, but the changes did fall into one of four categories we have called type $D$ (for net decrease in height; 57/82), type S (for same size after treatment; 13/82), type I (for net increase in height; $10 / 82$ ), and type $M$ (for miscellaneous; 2/82). One patient was lost to follow-up.

Type $D$. This pattern is characterised by a progressive diminution in tumour height immediately after therapy (Fig 2). In some cases the tumours remained stationary after shrinking and then shrank again. The maximal shrinkage seen was $8.1 \mathrm{~mm}$ in 29 months. In cases where the tumour diminished in size after reaching a plateau, the second regression began eight to 60 months after treatment. In these cases the magnitude of change was occasionally dramatic. One patient's tumour shrank $3 \mathrm{~mm}$ in two

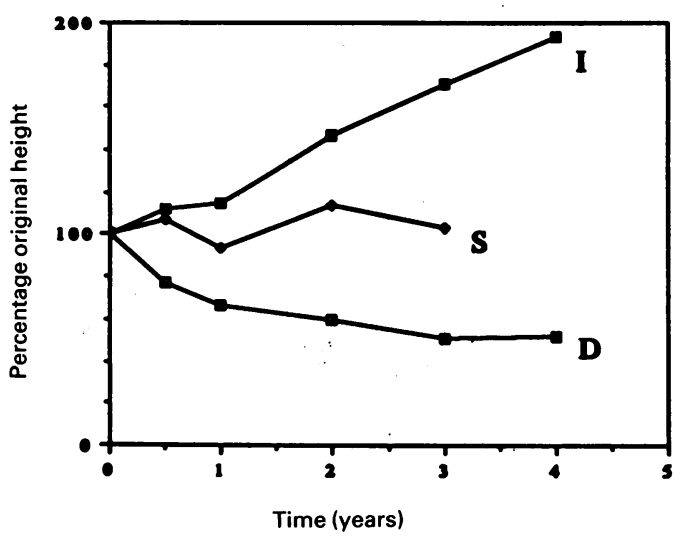

Figure 2: Percentage changes (normalised) for each of the three post-treatment types. 


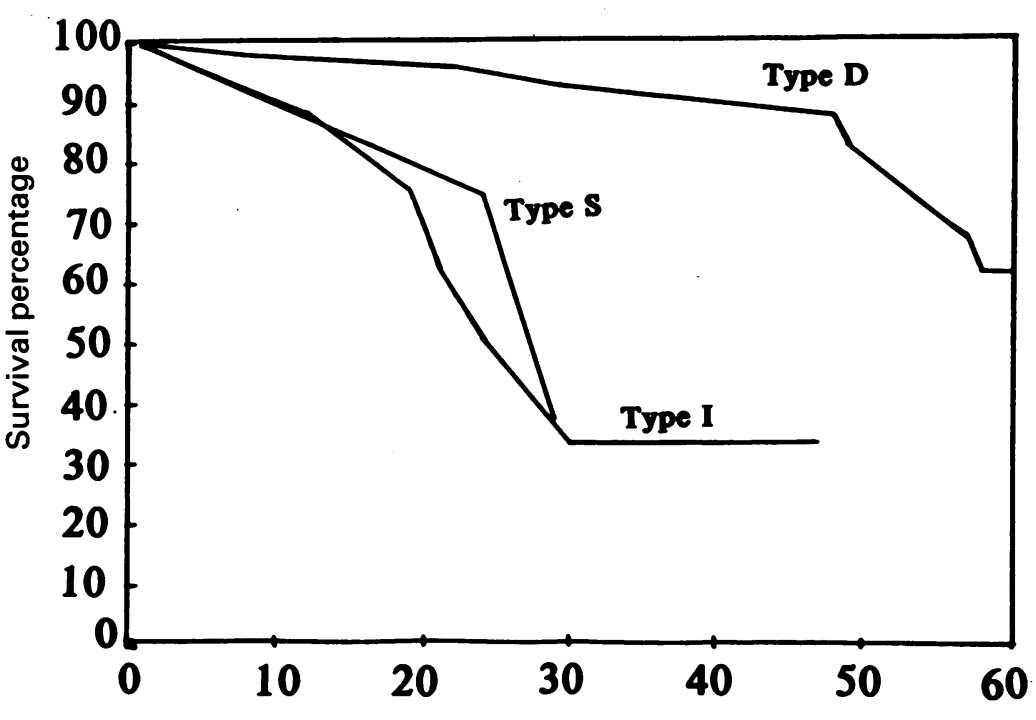

Time (months)

Figure 3: Life table analysis of survival for patients by tumour type. height after plaque radiotherapy for choroidal melanomas agree with those of some prior studies, disagree with some observations of other investigators, and may prove to be a useful classification which clinicians can use as a guideline in following up patients.

What happens to the size of uveal melanomas after plaque treatment? Although Stallard ${ }^{1}$ reported that the majority $(70 \%)$ of melanomas he treated with cobalt- 60 plaques became 'flat pigmented stippled' scars, he did not describe the time course of these changes. His observations were not made with the aid of ultrasonography and were largely based on the height measurement made with the direct ophthalmoscope.

Bedford, reporting on Stallard's cases and his own, noted that regression was frequently not complete for $1 \frac{1}{2}$ to 2 years after therapy. ${ }^{7}$ Long $e t$ $a l^{8}$ also demonstrated that melanomas treated with cobalt- 60 plaques showed little, if any, changes for four to six months after treatment.

Most of the recent observations on this subject come from two institutions. From Cornell, Coleman et al found that tumours showed a progressive diminution of height (measured by ultrasound) at 6,12 , and 18 months after treatment and that this magnitude of change was $53.9 \%$ for ATT B and $26.4 \%$ for ATT E at 18 months. ${ }^{3}$

In 1984 Cruess et al, ${ }^{2}$ reporting from the Wills Eye Hospital on their first 100 patients with uveal melanomas treated with cobalt -60 plaques, found that the 'average' posterior uveal melanoma did 'not regress rapidly to a flat, depigmented scar' but 'shrank slowly' to $50 \%$ of the thickness of the original tumour after 54 months. In the same paper the authors pointed out that there was no change in the maximal or minimal basal diameter of the tumour during 54 months of follow-up. ${ }^{2}$ Additional analysis of the same 100 patients revealed that $96 \%$ showed a regression and that only three melanomas increased in thickness after therapy. ${ }^{9}$

In another analysis of 17 patients from Wills who were followed up for at least three months the post-treatment rate of decrease in tumour thickness (\%/month) varied between 1.2 and $10 \cdot 5$. In no patient did the tumour grow after plaque therapy. ${ }^{10}$

We found that the mean post-treatment rate of decrease in large tumour thickness was: $2 \cdot 3 \%$ / month in the first six months, $1.5 \% /$ month in the second six months, $0.5 \% /$ month in the second year, and $0.9 \% /$ month between year 2 and year 4 . The changes for medium tumours were $1 \%, 2 \%$, $0.75 \%$, and $0.45 \%$ respectively.

The largest series published in which ultrasonic measurements were made was also from Wills, with an analysis of 159 patients treated between 1976 and 1980 . In this series the prognostic value of the regression rate was studied."

Our results generally agree with those of all the prior studies but have important features not discussed by others. We found that both the absolute and percentage decrease in tumour height were most pronounced in the large tumours and that the decrease in height tends to level off after $\mathbf{3 0}$ months. The average monthly decrease in tumour height for the first $\mathbf{4 8}$ months
Discussion

The results of this study of changes in tumour 
was $0.5 \mathrm{~mm}$ for large and $0.2 \mathrm{~mm}$ for medium tumours. The mean absolute change in tumour height after treatment in large tumours was $1 \cdot 8$ $\mathrm{mm}$ at six months and $5.5 \mathrm{~mm}$ at 48 months. For medium sized tumours these means are $0.9 \mathrm{~mm}$ and $1.9 \mathrm{~mm}$ respectively. When we actually superimposed each of the individual tumour responses, we could not find two tumours with exactly the same magnitude and time changes (Fig 1). This emphasises the individual responses of these tumours and should make clinicians wary of any discussion of 'average' changes.

When we looked for trends in height changes, we found that 80 of the 82 tumours fell into three categories. The most common (57 out of 82) of these was a progressive decrease in height after treatment (type D). The maximal shrinkage seen in this group was $8.1 \mathrm{~mm}$ in 29 months. In some patients the tumour got smaller, then remained stationary, and then finally shrank again 8 to 60 months after treatment. Only longer follow-up will reveal what ultimately happens to the size of these tumours.

The next commonest pattern seen was one where the tumour showed no response or remained exactly the same height following treatment (type $S$ ). These patients have been followed up for as long as 29 months. It is not known whether the tumour will eventually shrink or grow.

The least common, but perhaps the most important group, are those tumours which progressively increased in height (type I) after treatment. This increase continued until metastasis occurred, which is true for 5 out of 10 patients with this type to date.

-We found that age was an important predictor of survival. Patients older than 60 did less well than patients under 60 when analysed in a multivariate fashion. This is a feature which has previously been reported by others and deserves re-emphasis.

It was of great interest that the three radiation regression patterns correlated with the development of metastasis, in contrast to the prior data from Wills. At four years of follow-up a life table analysis showed more than $66 \%$ with type I were dead versus $12 \%$ with type D. In 1984 the Wills group reported that the 'rate and extent of regression of posterior uveal melanomas following cobalt-60 plaque radiotherapy are poor indicators of the ... subsequent development of clinical metastatic disease'. This was based on the first 100 patients treated there.

A further analysis of 159 patients from the same institution has been widely interpreted as showing that the tumours which most rapidly regressed have a greater chance of developing clinical metastasis. " In reality these authors showed that with univariate Cox modelling only the basal diameter at treatment correlated with survival (the larger the basal diameter, the greater the chance of developing metastasis). When multivariate Cox analysis was done, the most satisfactory model was one where the pretreatment basal diameter and the tumour thickness at 12 months after radiation were used." Neither the Wills study nor this one showed a correlation with survival when the percentage change in tumour thickness was studied at 3 months, 6 months, or 12 months after treatment.

While we believe that the local response to therapy does correlate with survival and that the patients who develop metastasis are more likely to be those whose tumour grows after therapy, we emphasise that clinical metastases also develop in patients in whom the tumour remains the same or decreased after plaque therapy. Preventing growth of the tumour may be defined as 'local control' but does not necessarily mean that no metastasis will develop. Similarly, a decrease in size of the eye tumour may be associated with the clinical development of metastasis.

There may be many reasons for the failure of a plaque to cause tumour regression. Some of those reasons may be known at the present time while others may not. Geographic 'miss', or poor placement of the plaque, low dose or dose rate, and the presence of radiation-insensitive cells may be among them. Since enucleation of melanomas has been implicated as a causative factor for the development of metastasis, it should be mentioned that of nine patients whose tumours grew after plaque therapy, seven whose eye was enucleated died, while two who refused enucleation also died.

1 Stallard HB. Radiotherapy for malignant melanoma of the choroid. Br F Ophthalmol 1966; 50: 147-55.

2 Cruess AF, Augsburger JJ, Shields JA, Brady LW, Markoe AM, Day JL. Regression of posterior uveal melanomas following cobalt-60 plaque radiotherapy. Ophthalmology 1984; 91 : 1716-9.

3 Coleman DJ, Lizzi FL, Silverman RH, et al. Regression of uveal malignant melanomas following cobalt-60 plaque. Retina 1985; 5: 73-8.

4 Cox DR. Regression models and life tables. $\mathcal{F} R$ Statistical Soc 1972; 34 (ser B): 187-220.

5 Kaplan EL, Meier P. Nonparametric estimation from incomplete observations. F Am Statistical Assoc 1958; 53:

6 Mantel N. Evaluation of survival data and two new rank order statistics arising in its consideration. Cancer Chemother Rep 966; 50: 163-70.

7 Bedford MA. The use and abuse of cobalt plaques in the treatment of choroidal malignant melanoma. Trans Ophthalmol Soc UK 1973;

8 Long RS, Galin MA, Rotman M. Conservative treatment of intraocular melanomas. Ophthalmology 1971; 75: 84-93.

9 Shields JA, Augsburger JJ, Brady LW, Day JL. Cobalt plaque therapy of posterior uveal melanomas. Ophthalmology 1982; 89: 1201-7.

10 Augsburger JJ, McNeary BT, von Below H, et al. Regression of posterior uveal malignant melanomas after cobalt plaque radiotherapy. Graefes Arch Clinc Exp Ophthalmol 1986; 224
397-400.

1 Augsburger JJ, Gamel JW, Shields JA, et al. Post-irradiation regression of choroidal melanomas as a risk factor from metastatic disease. Ophthalmology 1987; 94: 1173-7. 